 \\ HUDDERSFIELD
}

Mental Health and Learning Disabilities Research and Practice

Volume 3 Number 1 April 2006

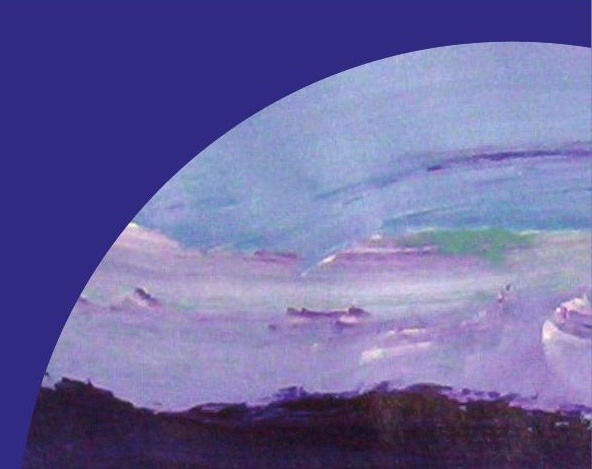




\title{
Pharmacological Treatment of Depression in Older People
}

\author{
Stephen Curran ${ }^{1}$, Andrew Byrne ${ }^{2}$, John Wattis ${ }^{3}$
}

${ }^{1}$ University of Huddersfield and South West Yorkshire Mental Health NHS Trust

${ }^{2}$ South West Yorkshire Mental Health NHS Trust

${ }^{3}$ University of Huddersfield 


\title{
Pharmacological Treatment of Depression in Older People
}

\author{
Stephen Curran, Andrew Byrne and John Wattis
}

\begin{abstract}
In the light of recent National Institute for Clinical Excellence (NICE) and Committee for the Safety of Medicines (CSM) guidance we discuss the importance of the diagnosis of depression in old age and review pharmacological interventions. An introductory section is followed by sections on each of the main antidepressant groups. This briefly describes their pharmacology and reviews research done specifically relevant to older people. Finally practical clinical applications are discussed.
\end{abstract}

\section{Introduction}

NICE guidance on management of depression is quite specific and detailed (see Box 1). According to NICE "watchful waiting" and "guided self help" are the best strategies for mild depression. For moderate or severe depression medication, psychological interventions and social support are appropriate. They can, in the first instance, be delivered in primary care. Where depression is treatment resistant, atypical, recurrent or psychotic or where there is "significant risk" specialist mental health teams should be involved using medication, complex psychological interventions and/or combined treatments. (In the experience of the authors this includes many older people with moderate and all with severe depression.) Finally, where there is risk to life or severe self-neglect crisis intervention and inpatient care with medication, combined treatments and ECT may be needed.

Box 1 Key points from NICE guidance on the management of depression (modified)

- Screening is justified in high risk sub groups, for example those with chronic disability or in hospital or residential care

- Guided self help (where the patient wants it) or "watchful waiting" with a further assessment within two weeks is the correct intervention for mild depression. Antidepressants are not recommended for this group.

- Short term psychological treatment specifically focused on depression is recommended for mild to moderate depression

- SSRI antidepressants are the first choice drugs for uncomplicated moderate depression but other drugs also make a major contribution particularly in more severe or resistant depression

- Patients should be fully informed about medication including discontinuation symptoms

- In severe depression the combination of cognitive behavioural therapy (CBT) with medication should be considered as it is more effective than either treatment alone.

- In recurrent depression continuation of antidepressants for at least two years and $\mathrm{CBT}$ both have a role 
It is essential that high quality psychological interventions are made available to older people (Everybody's Business, 2005). There is a need for research into the availability of these interventions but a description of them is beyond the scope of this review. A comprehensive account of Cognitive Behavioural Therapy (CBT) with Older Adults can be found in Laidlaw et al (2003).

Depression in later life is very similar to depression at other times of life and some have argued that there is insufficient evidence for a subtype of major depression in older people (Baldwin et al., 2002) though this is not a universally held view. However, ageing and other factors may alter the presentation of depression in later life. In particular older people are less likely to complain of sadness compared with younger patients and they are more likely to complain of physical symptoms, memory complaints and anxiety symptoms (Baldwin et al., 2002). In addition, depression in patients with dementia may lead to behavioural disturbance (Dwyer and Byrne, 2000).

Depression also has a significant impact on individual patients, their families and society more generally. In particular, depression is one of the leading causes of disability, it leads to a greater risk of hospitalisation as well as inappropriate bed use and prolonged hospitalisation; it is the single most important predictor of suicide. It also reduces compliance with medical treatments, reduces the patient's quality of life and is an independent predictor of mortality (Baldwin et al., 2002).

\section{Prevalence}

Depression is two to three times more prevalent than dementia and is the most common mental health problem amongst older adults. In community samples the prevalence of mild depression has been estimated to be $11 \%$ (Alexopoulous, 1992), rising to $22-33 \%$ in residential and nursing homes (Ames et al., 1988) and $45 \%$ in hospitalised older patients with physical illness (Koenig et al 1988). Depressive disorder, disability and dependency are all highly correlated and it is important to consider the diagnosis of depression in these settings.

\section{Aetiology}

A number of risk factors for depression have been identified. Genetic susceptibility is less important in depression when onset is in later life. Females are more susceptible to depression. A previous history of depression is an important predisposing factor and widows, widowers and divorcees are more susceptible to depression. Neurotransmitter changes are also well recognised in older people including reductions in noradrenalin and serotonin (Hindmarch, 2001). There are a number of other brain changes including cerebral atrophy but it is not entirely clear if this is related to depression. Deep white matter lesions and sub cortical grey matter lesions seem to be commoner in late onset depression (Curran and Wattis, 2004). Epidemiological studies have highlighted an association between hypertension and depression as well as other vascular risk factors including smoking, excessive alcohol consumption and hypotension. Personality factors are important predisposing factors particularly avoidant and dependent personality types. There is a strong association between 
disability (functional limitation) due to medical conditions and depression and especially with pain (Stahl and Briley, 2004). Being a carer of someone who is chronically ill is another important risk factor. Precipitating factors include life events such as bereavement, separation, acute physical illness, moving into residential care and chronic stress. A number of drugs can also cause or aggravate depression including beta-blockers, methyldopa, reserpine, clonidine, calcium channel blockers, digoxin, codeine, opioids, indomethacin, steroids, L-Dopa, amantadine, tetrabenazine, antipsychotics and benzodiazepines. Some factors can be protective including good medical care including general health, nutrition and physical fitness, good coping behaviours e.g. adaptive personality, capacity for confiding relationships and good social support including religious/spiritual beliefs (Baldwin et al., 2002).

\section{Association with physical illness}

Depression is an independent risk factor for a number of medical conditions including stroke (Jonas and Mussolino, 2000) and heart failure (Ariyo et al., 2000). Depressive symptoms also add to the disability from physical illness and are associated with physical decline (Penninx et al., 2000). A number of medical conditions increase the risk of depression including hypo/hyperthyroidism, Cushing's disease, hypercalcaemia, pernicious anaemia, cerebrovascular disease, Parkinson's disease, Alzheimer's disease, SLE, cancer, particularly of the lung and pancreas, and chronic infections such as brucellosis.

\section{Classes of antidepressants}

\section{Tricyclic antidepressants (TCAs)}

All these compounds are inhibitors of noradrenalin uptake with a variable potency of inhibiting serotonin uptake as well. They include amitriptyline, imipramine, dothiepin, doxepin, nortriptyline and lofepramine. Tricyclics can be subdivided into secondary (e.g. nortriptyline) and tertiary amines (e.g. imipramine and amitriptyline). Secondary amines are predominantly noradrenaline re-uptake inhibitors.

These drugs are generally well absorbed orally. They are lipophilic and widely distributed in the body. They are metabolised in the liver and some of the metabolites are active antidepressants. The major route of elimination is renal excretion. The lower rates of both hepatic metabolism and decreased renal clearance in this age group may reduce the dosage needed to achieve a given blood level. Some clinicians have recommended plasma level monitoring for TCAs in older patients but this is seldom done in clinical practice. Lofepramine has minimal anticholinergic properties and cardiac toxicity in older patients by comparison with older TCAs.

Drugs with a tertiary amine structure tend to produce more antagonism of $\alpha_{1}$ adrenergic receptors with subsequent hypotension, blockage of histamine (causing sedation) and cholinergic receptors (causing dry mouth, blurred vision, urinary retention, dizziness, tachycardia, memory impairment and at high and toxic doses, delirium). The tendency to produce orthostatic hypotension is a serious side-effect in the older patients leading to an increased risk of falls and limb fracture. 


\section{Clinical studies}

There are a limited number of double-blind, placebo-controlled trials of antidepressants involving older people and most of these are of TCAs (Mittman et al., 1997; Wilson et al., 2001). There is some evidence that TCAs are more effective than SSRIs in treating severe depression in older people (Navarro et al., 2001).

Patients commenced on TCAs often receive sub-therapeutic doses for inadequate periods of time compared with SSRIs (Donoghue and Hylan, 2001). However, a recent Cochrane review found that treatment of depression with low dose tricyclics is justified (Furukawa et al., 2004). In addition, a further Cochrane review of amitryptyline for older people found that it was at least as effective as other TCAs (in therapeutic doses) and newer compounds, especially SSRIs, but patients taking amitriptyline experienced a higher prevalence of side-effects (Guaiana et al., 2004).

\section{Selective serotonin reuptake inhibitors (SSRIs)}

SSRIs have a selective effect on serotonin reuptake, making them less likely to cause the side-effects encountered with TCAs. They include fluvoxamine, fluoxetine, paroxetine, sertraline, citalopram and escitalopram.

These drugs are rapidly absorbed from the gut and broken down into several metabolites (often with antidepressant activity) each with different half-lives. All SSRIs are extensively protein-bound. The plasma half-lives of fluvoxamine and fluoxetine are relatively unaffected by age whilst steadystate plasma concentration of paroxetine, sertraline and citalopram are higher in the elderly and elimination half-life is longer than for the same dose in adult patients (Baumann, 1998). Paroxetine has also some affinity for muscarinic receptors making it the most sedative SSRI. Administration of all SSRIs can augment TCA activity, as well as other drugs metabolised by the hepatic cytochrome P450 system. These interactions tend to be dose dependent.

All SSRIs are effective (65\% response rate) and well tolerated in older depressed patients (Baumann, 1998). They are relatively free of anticholinergic effects and cardio toxicity, and cause less postural hypotension than TCAs. Their main side-effects include headache, nausea, diarrhoea, insomnia, dizziness, and sexual dysfunction. All the SSRIs, with the exception of citalopram and sertraline, cause clinically relevant drugdrug interactions through their inhibitory effects on the cytochrome P450 system. Paroxetine, fluoxetine and fluvoxamine have inhibitory effects on cytochrome P450 isoenzymes that can lead to increased plasma levels of antipsychotics and TCAs (Anderson and Edwards, 2001). The half-lives of sertraline, citalopram, and paroxetine are prolonged in the older patients (Baumann, 1998) and lower starting doses of paroxetine (10mg/day) and perhaps citalopram may be needed. Elimination of citalopram, fluoxetine, sertraline and fluvoxamine are decreased in hepatic impairment and paroxetine elimination is reduced in renal impairment. 


\section{Clinical studies}

Wilson et al (2001) found that in older people with depression the number needed to treat for a therapeutic response to a TCA was 4 whereas for SSRls the number was 7 . However, only two trials met criteria for the analysis and both of these involved fluoxetine.

Sertraline is well tolerated in patients with major depression and acute MI or unstable angina. This study involved 369 patients many of whom were over 65 with a mean age of 57.1 years (Glassman et al. (2002). Sertraline was also effective and well tolerated in patients with co morbid medical illness in a randomised controlled trial (RCT) (with placebo) involving 752 older patients (Sheikh et al., 2004).

In a more recent long-term study looking at depression in older people, 254 patients were treated with sertraline and entered the treatment (8 weeks) and continuation phases (16-20 weeks) of the study. 113 patients entered the maintenance phase (100 weeks) and randomised into a double-blind, placebo controlled continuation/maintenance phase of approximately 2 years duration. No significant difference between the sertraline and placebo groups was found in the proportion of recurrences. The authors concluded that sertraline at therapeutic dosage does not provide significant protection against recurrence. However, very small numbers finished the trial (Wilson et al., 2003).

In a recent RCT involving 319 older patients with major depression, paroxetine controlled-release (CR) was compared with paroxetine immediate-release (IR) and placebo over 12 weeks. Both active treatments were more effective than placebo and responders were $72 \%(\mathrm{CR}), 65 \%$ (IR) and $52 \%$ for patients on placebo and these were significantly different (Rapaport et al., 2003).

Citalopram is well tolerated in older patients and in a review of 1344 patients from RCTs treated for a minimum of 6 weeks, bradycardia was significantly more likely in older people $(2.4 \%$ versus $0.2 \%)$ whereas gastrointestinal side-effects, sweating and headache were less prevalent in older patients (Barak et al., 2003). There have been no published reports of escitalopram in older people (Bielski et al., 2003).

The Committee on Safety of Medicines in 2004 issued general guidance on the use of SSRIs. The report examined SSRIs in both children and adults and focused particularly on suicidal behaviour and withdrawal reactions. In relation to adults the report concluded that "From the available clinical trial data, both published and unpublished, a modest increase in the risk of suicidal thoughts and self-harm for SSRIs compared with placebo cannot be ruled out. There is insufficient evidence from clinical trial data to conclude that there is any marked difference between members of the class of SSRIs or between SSRIs and other antidepressants with respect to their influence on suicidal behaviour". In relation to withdrawal reactions the report concluded that "All SSRIs may be associated with withdrawal reactions on stopping or reducing treatment. Paroxetine and venlafaxine seem to be associated with a greater frequency of withdrawal reactions than other SSRI" (CSM, 2004) 


\section{Serotonin and noradrenaline reuptake inhibitors (SNRIs)}

Venlafaxine selectively inhibits the uptake of serotonin and noradrenaline and in comparison with TCAs show no affinity for other neuroreceptors (Mendlewicz, 1995). No dosage adjustments are recommended for older people. Compared with SSRIs, venlafaxine affects the cytochrome P450 enzyme system relatively little. The most common side-effects include nausea, headache, dry mouth, dizziness, constipation and hypertension, although postural hypotension can be a problem in older people and should be monitored. To minimise risk of discontinuation symptoms, the dose of venaflaxine should be gradually tapered over a few weeks period. SSRIs and venlafaxine are also associated with hyponatraemia in older patients (Kirby et al., 2002).

\section{Clinical studies}

Venlafaxine is well tolerated (Danjou and Hackett, 1995) and shows similar efficacy to TCAs (Mahapatra and Hackett, 1997). There is evidence that the efficacy of venlafaxine is dose related and that at higher doses (>150mg daily) venlafaxine is more effective than SSRIs in major depression (Smith et al., 2002).

In a RCT comparing dothiepin and venlafaxine in 92 older patients with major depression, response to therapy in the venlafaxine group was $60 \%$ compared with $53 \%$ in the dothiepin group but these were not significantly different. Side-effects were very similar in the two groups and not significantly different (Mahapatra and Hackett, 1997).

In a 10-week randomised-controlled, double-blind trial of venlafaxine (up to $150 \mathrm{mg} /$ day) versus sertraline (up to $100 \mathrm{mg} /$ day) in 52 older nursing home patients with major depression, venlafaxine was less well tolerated but there were no significant differences in response to treatment (Oslin et al., 2003).

However, in a systematic review of eight RCTs, remission rates were significantly higher with venlafaxine compared with an SSRI in a series of studies involving over 2000 patients but the mean age in all groups was approximately 40 years (Thase et al., 2001). In addition, in a recent RCT involving eight older patients, venlafaxine $37.5 \mathrm{mg}$ bd had no significant effects on cognition or psychomotor performance compared with dothiepin (Trick et al., 2004).

Recent advice has been issued by the Committee on Safety of Medicines (2004) in relation to venlafaxine. In summary, venlafaxine should only be initiated by specialist mental health practitioners or GPs with a special interest in mental health and there should be appropriate supervision of patients. Venlafaxine should not be initiated in patients with heart disease e.g. cardiac failure, coronary artery disease, patients with ECG abnormalities including QT prolongation, in patients with electrolyte disturbances and in patients with hypertension. Patients currently taking and tolerating venlafaxine and getting clinical benefit should continue to the end of the course (CSM, 2004). 
Recently duloxetine became available. It is also a dual uptake inhibitor of both serotonin and noradrenaline with comparable affinities for both systems. A number of studies in younger patients have shown it to be effective with good safety and tolerability (Hudson et al., 2005) and as well as being effective and well tolerated in older people with depression (Nelson et al., 2005) but further work is needed.

\section{Monoamine oxidase inhibitors (MAOIs)}

Traditional MAOls include phenelzine, isoniazid and tranylcypromine. These bind non-selectively, either irreversibly or almost so, to both type A and type B monoamine oxidase enzymes to prevent the destruction of endogenous catecholamines (noradrenaline and dopamine) and the sympathomimetic amine tyramine. Because they lack selectivity, traditional MAOls can lead to serious side-effects when foods rich in tyramine or other amines are eaten. In addition, these drugs have significant interactions with other drugs that seriously limit their use compared with other antidepressants. However, they may have a place in the treatment of resistant depression (Georgotas and McCue, 1989), but they are seldom used as first line drugs. There is no good quality data specifically in relation to depression in older people.

\section{Reversible inhibitor of monoamine oxidase (RIMA)}

These drugs preferentially inhibit monoamine oxidase $A$ but the inhibition is reversible. Moclobemide is effective and well tolerated and may be a useful alternative for depressed patients with prominent anxiety symptoms (Tourigny-Rivard, 1997). In addition, it may enhance cognition in dementia with depressive symptoms (Roth et al., 1996). It lacks anticholinergic and cardio toxic side-effects but it can cause insomnia and nausea. Drug interactions can occur with sympathomimetics and opiates but unlike traditional MAOls it requires little or no dietary restrictions. Bonnet (2003) has recommended combining moclobemide with another antidepressant e.g. clomipramine or an SSRI for treatment resistant depression. However, these combinations are not without risks.

\section{Noradrenaline and serotonin synaptic antagonist (NaSSA)}

Mianserin and mirtazapine are both potent antagonists at $\alpha_{2}$-autoreceptors. This leads to an increase in noradrenaline release. These drugs have low affinity for muscarinic, cholinergic and dopamine receptors resulting in reduced side-effects. Mianserin has fallen out of favour because of a small risk of agranulocytosis necessitating blood monitoring and there is very little data in older patients. Mirtazapine has effects on both the noradrenergic and sertonergic systems. It enhances central noradrenergic and $5 \mathrm{HT}_{1}$ receptor mediated serotonergic neurotransmission. It is also an antagonist at $\alpha_{2}$ presynaptic autoreceptors and also blocks $5 \mathrm{HT}_{2}$ and $5 \mathrm{HT}_{3}$ receptors. This results in increased noradrenergic release. Changes in pharmacokinetics with age are considered minor (Timmer et al., 1996). The main side-effects of mirtazapine include sedation and weight gain and less commonly mania, convulsions, paraesthesia and reversible agranulocytocis. 


\section{Clinical studies}

Mirtazapine (maximum $35 \mathrm{mg}$ ) has been compared with trazodone (maximum $280 \mathrm{mg}$ ) in a placebo-controlled, double-blind trial over 6 weeks involving 150 older patients with major depression. Significant improvements in depression were observed with both active compounds from week 2 compared with placebo. However, both drugs had significantly higher levels of somnolence and dry mouth and trazodone dizziness, and blurred vision (Halikas, 1995).

A similar study compared mirtazapine with amitriptyline (30-90 mg) in 115 older depressed patients in a double-blind, multi-centre study. Similar clinical benefit was observed with both drugs but the Clinical Global Impression (CGI) was statistically better in the amitriptyline group. Sideeffects were noted to be similar in both groups (Hoyberg et al., 1996)

In a recent RCT, mirtazapine was compared with paroxetine over 8 weeks in 255 older patients with major depression. Mirtazapine had a significantly earlier onset of action and was better tolerated (Schatzberg et al., 2002).

\section{Noradrenaline re-uptake inhibitors (NARIs)}

Recent evidence suggests that reboxetine is effective in older people with depression but causes less hypotension and fewer serious side-effects than TCAs (Katona et al., 1999). However, renal clearance is reduced in older patients (Bergmann et al., 2000). The pharmacokinetics of reboxetine are linear following both single and multiple oral doses and the plasma halflife is approximately 12 hours. Metabolism is mainly via the liver with only $10 \%$ cleared by the kidneys but plasma concentrations are increased in older patients.

In a prospective, uncontrolled, multi centre study of reboxetine involving 160 older patients with major depression over 52 weeks, 104 patients completed the study. The proportion who were rated as either "much" or "very much improved" were $15.1 \%$ at 2 weeks and $88 \%$ at 6 weeks with $95.2 \%$ at 52 weeks. The drug was well tolerated although nausea $(11.9 \%)$ and headache (10\%) were relatively common (Aguglia, 2000). However, because of these side-effects some authors have recommended that older patients should be started on $4 \mathrm{mg} /$ day in two divided doses (Bergmann et al., 2000). Controlled trials in older people are still needed.

\section{Which antidepressant is best for older people?}

There is no clear answer to this question. However, in a review of 18 metaanalyses of different antidepressants mainly involving younger patients, the main finding was a lack of major difference in efficacy between the newer and older antidepressants. Venlafaxine was found to be superior to SSRIs and SSRIs better tolerated. Interestingly, in older patients dothiepin was found to be better tolerated than SSRIs (Anderson, 2001). In addition, in a recent Cochrane review (Wilson et al., 2004) TCAs, SSRIs and MAOls were reported to be effective in the treatment of older community patients and inpatients with severe physical illness. At least six weeks of antidepressant treatment was recommended to achieve optimal therapeutic effect. 
Antidepressants do not help everyone with depression. Although much has been written about how to identify responders this is still difficult in clinical practice and "trial and error" is still largely the norm.

\section{Practical prescribing}

Although depression can be successfully treated it is frequently missed and treated with suboptimal doses of antidepressants for inadequate periods of time. The treatment of depression in older people invariably needs an integrated approach with pharmacological, social and psychological approaches working together. Patient education is also an important component of successful treatment and it is essential to work closely with families (Wilson et al., 1999).

The optimal treatment of depression in later life is crucial and requires appreciation of a number of factors such as co-morbidity, polypharmacy, altered drug kinetics, variable treatment response and increased predisposition to side effects. Older people need to be thoroughly assessed (Curran et al., 2005) before commencing antidepressants due to the increased incidence of physical illnesses, changes in pharmacokinetics and increased sensitivity to side-effects. It is also an important opportunity to develop a good rapport with patients to improve understanding of and compliance with medication. Despite the NICE (2004) recommendation that SSRIs are recommended and (particularly on the basis of cost-benefit, fluoxetine or citalopram) there is no single "first line" antidepressant and individual prescribing should take into account a number of factors including efficacy, safety, tolerability, real-world efficacy and economic value (Mendlewicz, 2001). Regular review is also important to monitor compliance, response, side-effects and physical health.

\section{Acute treatment}

Approximately $70 \%$ of patients will respond to an antidepressant (Stahl, 2000) but this can be lower in older people with rates of $40-60 \%$ being more typical (Williams et al., 2000). However, only approximately $50 \%$ of patients with major depression achieve remission with their first antidepressant (Pridmore and Turnier, 2004). In a recent systematic review of 186 RCTs mainly involving younger people, amitriptyline was found to be less well tolerated but slightly more patients recovered compared with alternative antidepressants (Barbui and Hotopf, 2001).

Several studies have shown that the onset of therapeutic action may take longer in older people and that optimum benefit can take 8-12 weeks after commencing treatment (Georgotas and Cooper, 1989). When initial treatment with a tricyclic has been ineffective a second antidepressant may take 5-6 weeks to have an effect (Flint and Rifat, 1996). 'Start low; go slow' is a useful guide when prescribing in older people but with the aim of reaching therapeutic dosages. Older patients are at high risk of recurrence following a depressive episode, with up to a $70 \%$ risk of recurrence within two years of remission (Flint, 1992). Therefore, after a successful response, continuation of antidepressant medication may be needed for at least two years. 


\section{Continuation treatment}

How long should an antidepressant be continued for? Antidepressant drug treatment should be continued for at least 12 months after remission in the elderly (OADIG, 1993; Anderson et al., 2000). If antidepressant treatment is stopped within 6-12 months and substituted with a placebo, approximately $50 \%$ of patients will relapse. In addition, if treatment is continued for 12 months, $90 \%$ will continue to respond. The risk is greater if the patient has had a number of previous episodes e.g. $1(<50 \%), 2(50-90 \%)$ and 3 $(>90 \%)$ (Stahl, 2000). Continuation drug therapy reduces the risk of relapse after remission. It is not a fixed period but in older people a 12-month period of continuation with antidepressants is recommended in contrast to 6 month period for younger patients (Baldwin et al., 2002). However, patients with recurrent depression should be treated for two years (or more). For patients with delusional depression on anti-psychotic medication, it is recommended that this be continued for 6 months before being tailed off.

\section{Maintenance treatment}

When should maintenance therapy be used? This would usually be if there has been two or more previous episodes for younger patients but one or more previous episodes for older patients (Stahl, 2000).

The dose of antidepressant should be with the same dose as used to treat the acute episode (Anderson et al., 2000). Older patients taking dothiepin over a two- year period are 2.5 times less likely to relapse compared with those taking placebo (OADIG, 1993). A recent study has shown that maintenance therapy with either nortriptyline or paroxetine was effective in $80-90 \%$ of older patients over an 18-month period (Reynolds et al., 1999).

In older patients, citalopram prevented recurrence over a period of 1-2 years suggesting that protection is not confined to TCAs (Appelberg, 2002). However, Wilson et al. (2001) did not find any benefit from sertraline in prophylaxis. Possibly the dose used was too small. However, this study cautions against assuming all antidepressants are equally effective in prevention. The case for long-term antidepressant treatment needs to be balanced against adverse effects, which in the case of older antidepressants can include troublesome weight gain and cardiovascular disturbance.

Lithium salts are also widely used for the prophylaxis of recurrent depression despite lack of adequate studies in older patients. A small prospective study by Abou-Saleh and Coppen (1983) found that an older sub-group benefited from lithium prophylaxis. However, clearance of lithium is reduced in older patients, so half the adult dosage may be required and maintenance levels of $0.5 \mathrm{mmol} / /$ have been suggested. Lithium should be used cautiously with SSRIs because of the risk of serotoninergic neurotoxicity.

\section{Management of treatment resistant depression}

Treatment resistance is reported in up to $40 \%$ of older patients with major depression (Flint, 2002). There is little evidence to guide the management of depression that has failed to respond to a course of an antidepressant 
and further research is needed to inform clinical practice. A review of 29 studies concluded that $35-60 \%$ of older patients showed a good recovery from a first depressive episode (Angst, 1997). However, research on treatment resistant depression in older people is hampered in part by lack of an agreed definition.

The definition of treatment resistant depression can be confusing. Some have suggested that it is failure to respond to one effective medication at a therapeutic dosage and for an adequate duration (Cowen, 1998). Renwick (1985) has suggested that it is the failure to respond to an adequate trial of two antidepressants or an antidepressant and/or ECT. Thase and Rush (1997) elaborated the definition further by developing a staging system of treatment resistance based on types of failed treatment.

The first step is to review the diagnosis and ensure that an organic cause has not been missed. Further investigations may be necessary such as an ECG, Chest X-ray, EEG or CT head scan. Severe deep white matter lesions on a brain MRI scan (O'Brien and Barber, 2000) and prefrontal dysfunction may predict a poor response to antidepressant treatment (Kalayam and Alexopoulous, 1999). Depression in patients with Alzheimer's disease is more likely to resolve spontaneously without requiring intensive antidepressant treatment compared with patients with vascular dementia who are more likely to have persistent and treatment resistant depressive symptoms ( $\mathrm{Li}$ et al., 2001). The presence of a silent cerebral infarction also reduces treatment response to antidepressant treatment (Yamashita, 2000).

Where there are maintaining social or environmental factors, psychological therapies may be beneficial as an adjunct to pharmacological treatment. A recent meta-analysis of psychological outreach programmes demonstrated a large effect size (0.7) in depressed older patients (comparable to effect sizes found in younger adults). Cognitive behavioural therapy was found to have a larger effect size than other therapies but also had higher drop out rates (Cujpers, 1998).

It is also important to ensure treatment adequacy in terms of duration and compliance. This has been called a stepped-care approach, meaning that each step of treatment follows a logical sequence rather than a chaotic mishmash (Guscott and Grof, 1991). This has been reinforced in a study by Flint \& Rifat (1996) in which over $80 \%$ of patients recovered if a logical process is adopted, for example starting with a trial of antidepressant monotherapy and eventually moving up to ECT.

\section{Some of the specific drug-related approaches include:}

\section{Change the dose}

This usually though not always means increasing the dose. This may be beneficial for TCAs and venlafaxine but there is little evidence that increasing the dose significantly alters the outcome in the case of SSRIs. At higher doses ( $>150 \mathrm{mg}$ daily) venlafaxine may be more effective than SSRIs in major depression (Einarson et al., 1999). Blood pressure monitoring is recommended when patients are taking high doses of venlafaxine (>200mg daily). 


\section{Prolong the length of treatment}

Here the therapeutic trial is continued beyond what is usually considered an 'adequate' period of time. There is evidence that an extension to nine weeks or even longer may improve recovery from depressive episode in older patients (Georgotas and McCue, 1989), although not if there has been no improvement in the first four weeks (Mottram et al., 2002).

\section{Switching antidepressant class}

Switching between classes of antidepressants has been shown to have modest benefit, although there is little systematic evaluation. The most popular strategy is a change from one antidepressant to another from a different class: for example from a TCA to an SSRI, or vice versa (Akiskal, 1985). Venlafaxine showed some promise in resistant depression but evaluation is mostly confined to clinical experience (Bowskill and Bridges, 1997), or open trials (Nierenberg et al., 1994). It has been estimated that the overall success rate for class switching is about $50 \%$ (Baldwin, 1996).

\section{Lithium augmentation}

Following failure of two classes of antidepressants, traditionally, the next step would be lithium augmentation. As yet there have been no randomised-controlled trials of lithium augmentation in older patients. Flint and Rifat (1994), in an open prospective study, showed a $23 \%$ response rate. They also found that $50 \%$ of older patients experienced side-effects including polydipsia, polyuria, tremor, dry mouth and nausea. Fahy and Lawlor, (2001) followed up a small cohort of older depressed patients who had their long-term lithium augmentation therapy discontinued for varying reasons, 52\% relapsed and the longer the length of lithium treatment prior to discontinuation the poorer the outcome. The time needed to judge response to lithium augmentation is approximately to 2-3 weeks (Cowen, 1998).

Austin et al. (1991) conducted a meta-analysis of five small double-blind trials and found that $18 / 50$ of patients augmented with lithium responded compared with $6 / 49$ of those who were given placebo. In another study (Hoencamp et al., 2000) venlafaxine and lithium augmentation was studied in an open trial in out-patients aged 18-70. At the end of 7-weeks study, $35 \%$ of the patients showed a more than $50 \%$ reduction in depressive symptoms and the combination was well tolerated. Other augmentation approaches include use of neuroleptics, anticonvulsants, thyroid hormone and L-tryptophan but the evidence-base is too weak to make firm recommendations for older patients.

\section{Combination therapies}

MAOls and TCAs combinations (+ or - lithium) has been used for decades in resistant cases. A review by Cowen (1998) demonstrated how open studies in younger adults have shown failure of each type of drug individually, but with good results when combined. Starting at very low dose, adding in the MAOI and not using imipramine or clomipramine is thought to lessen the risks of hazardous side effects and interactions. 
There is no evidence for this combination in older people and both MAOls and TCAs are being used less compared with several years ago.

Other antidepressant combinations that have been tried include TCAs and SSRIs. Again there are no controlled trials. Cowen (1998) suggests that due to the high risk of side-effects, drugs such as clomipramine or venlafaxine (with potent serotonin as well as noradrenaline reuptake inhibition in one medication), may be as effective as TCAs and SSRIs combined. There may be a role for low dose trazodone to augment SSRIs but this may just help the sleep disturbance and further studies are necessary.

A meta-analysis of tri-iodothyronine augmentation of TCA in mixed age groups showed a response rate of $8 \%(N N T=13)$ (Aronson et al., 1996). There are no studies comparing other classes of antidepressants. It should be used cautiously if there is co-existing cardiovascular disease. In adults, L-tryptophan has been shown to enhance the combinations of lithium-MAOI and lithium-clomipramine (Hale et al., 1987). Again there are no trials in older patients. With the risk of developing eosinophilic myalgic syndrome and possible serotonin syndrome with MAOls and SSRIs, this combination needs to be used with caution. There is no longer a need to register with the Optimax (tryptophan) information and clinical support (OPTICS) unit. Pindolol, a beta blocker with 5- $\mathrm{HT}_{1 \mathrm{~A}}$-receptor antagonist, had been shown in open studies to augment antidepressants. However, a small doubleblind, placebo-cross over trial showed no difference to placebo (Moreno et al., 1997). Dexamethasone (Bodani et al., 1999), clonazepam (Morishita and Aoki, 1999), oestrogen (Stahl, 1998), and methylphenidate (Emptage et al., 1996) have also all been tried but require further research.

Individual clinicians will invariably have their "preferred" combination of antidepressants e.g. mirtazapine and clomipramine and mirtazapine plus an SSRI but the evidence base for these is very poor. In addition, these combinations require increased monitoring and are not without risks.

\section{Electroconvulsive therapy (ECT)}

ECT can be used at any stage alongside the pharmacological management of treatment resistant depression. It should be noted that SSRIs may increase seizure length in ECT compared with TCA and may also increase the risk of post-treatment seizures (Curran, 1995). Shapira et al. (1998) have shown that the rate of response is more rapid when ECT is given three times a week but memory impairment is more common and suggested that the optimum schedule for bilateral ECT in older patients is twice weekly unless clinical indications require a more rapid antidepressant effect. Unilateral ECT may have an advantage with respect to cognitive side effects but Krystal et al (1998) suggest that moderately suprathreshold unilateral ECT may not be as effective as bilateral ECT for older patients. $\mathrm{He}$ found that older patients were more likely to have a rise in seizure threshold and to be non-responders to unilateral ECT.

\section{Discontinuing treatment}

Antidepressants need to be discontinued slowly (weekly decrements) as all have the potential to cause a withdrawal syndrome (Hoencamp et al., 
2000). Typical withdrawal symptoms are anxiety, agitation, insomnia, dizziness, low mood, paraesthesia, nausea and diarrhoea. When changing from one antidepressant to another, cross tapering is recommended.

\section{Conclusions}

Depression is common and if not treated results in high morbidity and mortality. Depression in older people generally has a good prognosis. It is therefore important to diagnose the condition and treat systematically. When an antidepressant is indicated, it should be chosen on an individual basis and used at a therapeutic dose for an adequate period. The choice of antidepressant will depend on a number of factors including previous response to treatment, concurrent physical illness and other medications. Pharmacological treatment also needs to be combined with psychosocial interventions. However, further well-planned research is needed into the pharmacological management of depression, especially treatmentresistant, in older people. 


\section{References}

Abou-Saleh, M.T. \& Coppen A. 1983. The prognosis of depression in old age: the case for lithium therapy. British Journal of Psychiatry,; 170, 285287.

Aguglia, E. 2000. Reboxetine in the maintenance therapy of depressive disorder in the elderly: a long-term open study International Journal of Geriatric Psychiatry, 15, 784-93..

Akiskal, H.S. 1985. An approach to chronic and 'resistant' depressions: evaluation and treatment. Journal of Clinical Psychiatry,; 46, 32-36.

Alexopoulous, G.S. 1992. Geriatric depression reaches maturity. International Journal of Geriatric Psychiatry, 7, 305-306.

Ames, D., Ashby, D., Mann, A.H. \& Graham, N. 1988. Psychiatric illness in elderly residents of part 3 homes in one London borough: prognosis and review. Age and Ageing, 17(4), 249-256.

Anderson, I. \& Edwards, J. 2001. Guidelines for choice of selective serotonin reuptake inhibitor in depressive illness. Advances in Psychiatric Treatment, , 7, 170-180.

Anderson, I.M., Nutt, D.J., \& Deakin, J.F.W. 2000. Evidence-based guidelines for treating depressive disorders with antidepressants; a revision of the 1993 British Association for Psychopharmacology guidelines. Journal of Psychopharmacology, 14(1), 3-20.

Anderson, I.M. 2001. Meta-analytical studies on new antidepressants. British Medical Bulletin, 1, 161-178.

Angst, J. 1997. A regular review of long term follow-up of depression. British Medical Journal, 315, 1143-1146.

Appelberg, B. 2002. Long-term citalopram prevents recurrent depression in the elderly and is well tolerated. Evidence Based Mental Health, 6(1):24.

Ariyo, A.A., Haan, M., Tangen, C.M. et al. 2000. Depressive symptoms and risks of coronary heart disease and mortality in elderly Americans. Circulation, 102, 1773-1779.

Aronson, R., Offman, H.J., Joffe R.T. \& Naylor, C.D. 1996. Triiodothyronine augmentation in the treatment of refractory depression. A meta-analysis. Archives of General Psychiatry, 53, 842-848.

Austin, M.P.V., Souza, F.G.M. \& Goodwin, G.M. 1991. Lithium augmentation in antidepressant-resistant patients: a quantitative analysis. British Journal of Psychiatry, 159, 510-14.

Baldwin, R.C. 1996. Treatment resistant depression in the elderly: a review of treatment options. Reviews in Clinical Gerontology, 6, 343-348. 
Baldwin, R.C., Chiu, E, Katona, C, \& Grahaam, N. 2002. Guidelines on Depression in Older People: practising the evidence. Martin Dunitz, London.

Barak, Y., Swartz, M., Levy, D. et al. 2003. Age-related differences in the side-effect profile of citalopram. Progress in Neuropsychopharmacology and Biological Psychiatry, 27(3), 545-548.

Barbui, C. \& Hotopf, M. 2001. Amitriptyline versus the rest; still the leading antidepressant after 40 years of randomised controlled trials. British Journal of Psychiatry, 178, 129-144.

Baumann, P. 1998. Care of depression in the elderly: comparative pharmacokinetics of SSRIs. International Clinical Psychopharmacology, 13 (suppl.5), 35-43.

N. F. N. Bergmann, J.F., Laneury, J.P., Duchene, P., Fleishaker, J.C., Houin, G \& Segrestaa, J.M. 2000. Pharmacokinetics of reboxetine in healthy, elderly volunteers. European Journal of Drug Metabolism \& Pharmacokinetics, 25(3-4), 195-198.

Bielski, R., Ventura, D. \& Chung-Chi C. 2003. A double-blind comparison of escitalopram with venlafaxine $\mathrm{XR}$ in the treatment of major depressive disorder. $16^{\text {th }}$ Congress of the European College of Neuropsychopharmacology, Prague, 20-24, September 2003.

Bodani, M. Sheehan, B. \& Philpot M. 1999. The use of dexamethasone in elderly patients with antidepressant-resistant depressive illness. Journal of Psychopharmacology, 13(2), 196-197.

Bonnet, U. 2003. Moclobemide; therapeutic use and clinical studies. CNS Drug Reviews, 9(1), 97-140.

Bowskill, R.J. \& Bridges, P.L. 1997. Treatment-resistant affective disorders. British Journal of Hospital Medicine, 57, 171-172.

Committee on Safety of Medicines 2004. Report of the CSM Expert Working Group on the Safety of Selective Serotonin reuptake Inhibitors Antidepressants, December 2004, www.mhra.gov.uk

Committee on Safety of Medicines 2004. Safety of Selective Serotonin Reuptake Inhibitor Antidepressants. www.mhra.gov.uk

Cowen, P.J. 1998. Pharmacological management of treatment-resistant depression. Advances in Psychiatric Treatment,,4, 320-327.

Cujpers, P. 1998. Psychological outreach programmes for the depressed elderly: A meta-analysis of effects and dropout. International Journal of Geriatric Psychiatry, 13(1), 41-48.

Curran, S. 1995. Effect of paroxetine on seizure length during electroconvulsive therapy. Acta Psychiatrica Scandinavica, 12(2), 239-240. 
Curran, S. \& Wattis, J.P. 2004. Psychopharmacology in the elderly. In: Seminars in Clinical Psychopharmacology (Ed. D.J King), $2^{\text {nd }}$ Edition, Gaskell, London, 447-484.

Curran, S, Nightingale, S. \& Wattis, J.P. 2005. Practical issues in assessing and prescribing psychotropic drugs in older people. In: Practical Old Age Psychopharmacology; A Multi-Professional Approach, S Curran and R Bullock (Eds.), Radcliffe Medical, Oxford, 47-72.

Danjou, P. \& Hackett D. 1995. Safety and tolerance profile of venlafaxine. International Clinical Psychopharmacology,, 10 (Suppl.2), 15-20.

Donoghue, J. \& Hylan, T.R. 2001. Antidepressant use in clinical practice; efficacy versus effectiveness. British Journal of Psychiatry, 179 (Suppl.42), 9-17.

Dwyer, M. \& Byrne, G.J. 2000. Disruptive vocalisation and depression in older nursing home residents. International Psycho geriatrics, 12, 463-471.

Einarson, T.R., Arikian, S.R,. Casciano, J. \& Doyle, J.J. 1999. Comparison of extended release Venlafaxine, selective serotonin re-uptake inhibitors, and tricyclic antidepressants in the treatment of depression: a metaanalysis of randomised controlled trials. Clinical Therapeutics, 21, 296-308.

Emptage, R.E., Semla, T.P., Gonzales, L. \& Larouche, M. 1996. Depression in the medically ill elderly: A focus on methylphenidate. Annals of Pharmacotherapy, 30(2), 151-157.

Everybody's Business. Integrated mental health service for older adults: a service development guide 2005. Care Services Improvement Partnership/ Department of Health, London: www.everybodysbusiness.org.uk.

Fahy, S. \& Lawlor BA. 2001. Discontinuation of lithium augmentation in an elderly cohort. International Journal of Geriatric Psychiatry, 16(10), 10041009.

Flint, A.J. 2002. Treatment-resistant depression in late life. CNS Spectrums, 7(10), 733-738.

Flint, A.J. \& Rifat, S.L. 1996. The effect of sequential antidepressant treatment on geriatric depression. Journal of Affective Disorders, 36, 95105.

Flint, A J. 1992. The optimum duration of antidepressant treatment in the elderly. International Journal of Geriatric Psychiatry, 7, 617-619.

Flint, A.J., Rifat, S.L. 1994. A prospective study of lithium augmentation in antidepressant-resistant geriatric depression. Journal of Clinical Psychopharmacology, 14, 353-356.

Furukawa, T., McGuire, H. \& Barbui C 2004. Low dosage tricyclic antidepressants for depression. Cochrane Review 3,. 
Georgotas, A. \& McCue, R. 1989. The additional benefit of extending an antidepressant trial past seven weeks in the depressed elderly. International Journal of Geriatric Psychiatry, 4, 191-195.

Georgotas, A.M.R., Cooper, T. 1989. Factors affecting the delay of antidepressant effect in responders to nortriptyline and phenelzine. Psychiatry Research, 28, 1-9.

Glassman, A.H., O'Connor, C.M., Califf, R.M., Swedberg, K. et al. 2002. Sertraline treatment of major depression in patients with acute $\mathrm{Ml}$ or unstable angina. Journal of the American Medical Association, 288(6), 701709.

Guaiana, G., Barbui, C. \& Hotopf, M. 2004. Amitriptyline versus other types of pharmacotherapy for depression. Cochrane Review 3.

Guscott, R. \& Grof. P. 1991. The clinical meaning of refractory depression: a review for the clinician. American Journal of Psychiatry, 148, 695-704.

Hale, A, Proctor, A.W. \& Bridges, P.K. 1987. Clomipramine, tryptophan and lithium in combination for resistant endogenous depression seven case studies. British Journal of Psychiatry, 151, 213-217.

Halikas, J.A. 1995. Org 3770 (mirtazapine) versus trazodone; a placebo controlled trial in depressed elderly patients. Human Psychopharmacology,; 10, 125-133.

Hindmarch, I. 2001. Expanding the horizons of depression; beyond the monoamine hypothesis. Human Psychopharmacology, 16(3), 203-218.

Hoencamp, E., Haffmans, P.M.J., Dijken, W.A., et al. 2000. Lithium augmentation of venlafaxine: an open-label trial. Journal of Clinical Psychopharmacology, 20, 538-543.

Hoyberg, O.J., Maragakis, B., \& Mullin, J. et al. 1996. A double-blind multicentre comparison of mirtazapine and amitriptyline in elderly depressed patients. Acta Psychiatrica Scandinavica, 93, 184-190.

Hudson, J.I., Wohlreich, M.M., Kajdasz, D.K. et al. 2005. Safety and tolerability of duloxetine in the treatment of major depressive disorder; an analysis of pooled data from eight placebo-controlled clinical trials. Human Psychopharmacology, 20, 327-341.

Jonas, B.S. \& Mussolino, M. E. 2000. Symptoms of depression as a prospective risk factor for stroke. Psychosomatic Medicine, 62, 463-471.

Kalayam, B. \& Alexopoulos, G.S. 1999. Prefrontal dysfunction and treatment response in geriatric depression. Archives of General Psychiatry, 56(8), 713-718.

Katona, C., Bercoff, E., Chiu, E., Tack, P., Versiani, M. \& Woelk, H. 1999. Reboxetine versus imipramine in the treatment of elderly patients with depressive disorders; a double-blind randomised trial. Journal of Affective Disorders, 55, 203-213. 
Kirby, D., Harrigun, S. \& Ames, D. 2002. Hyponatraemia in elderly psychiatric patients treated with SSRIs and venlafaxine: a retrospective controlled study in an in-patient unit. International Journal of Geriatric Psychiatry,; 17, 231-7.

Koenig, H.G., Meador, K. G., Cohen, H.J. \& Blazer, D. 1988. Depression in elderly hospitalised patients with medical illness. Archives of Internal Medicine, 148, 1929-1936.

Krystal, A.D., Coffey, C.E., Weiner, R.D. \& Holsinger, T. 1998. Changes in seizure threshold over the course of electroconvulsive therapy affect therapeutic response and are detected by ictal EEG ratings. Journal of Neuropsychiatry \& Clinical Neurosciences, 10(2), 178-186.

Laidlaw, K, Thompson, L.W., Dick-Siskin, L. \& Gallagher-Thompson, D. 2003. Cognitive Behaviour Therapy with Old People. John Wiley \& Sons, Chichester

Li, Y.S., Meyer, J.S. \& Thornby, J. 2001. Longitudinal follow-up of depressive symptoms among normal versus cognitively impaired elderly. International Journal of Geriatric Psychiatry, 16(7), 718-727.

Mahapatra, S.N. \& Hackett, D. 1997. A randomised, double-blind, parallelgroup comparison of venlafaxine and dothiepin in geriatric patients with major depression. International Journal of Clinical Practice, 51(4). 209-213.

Mendlewicz, J. 1995. Pharmacologic profile and efficacy of venlafaxine. International Clinical Psychopharmacology, 10 (Suppl.2), 5-13.

Mendlewicz, J. 2001. Optimising antidepressant use in clinical practice; towards criteria for antidepressant selection. British Journal of Psychiatry, 179 (Suppl. 42), 1-3.

Mittman, N., Herrmann, N., Einarson, T.R. et al. 1997. The efficacy, safety and tolerability of antidepressants in late life depression: a meta-analysis. Journal of Affective Disorders, 46, 191-217.

Moreno, F.A., Gelenberg, A.J., Bachar, K. \& Delgado, P.L. 1997. Pindolol augmentation of treatment-resistant depressed patients. Journal of Clinical Psychiatry, 58(10), 437-439.

Morishita, S., Aoki, S. 1999. Clonazepam in the treatment of prolonged depression. Journal of Affective Disorders, 53(3), 275-278.

Mottram, P, Wilson, K.C.M., Ashworth, L. \& Abou-Saleh, M. 2002. The clinical profile of older patients' response to antidepressants - an open trial of sertraline. International Journal of Geriatric Psychiatry, 17, 574-578.

Navarro, V., Gasto, C., Torres, .X, Marcos, T. \& Pintos, L. 2001. Citalopram versus nortriptyline in late-life depression: A 12 week randomized singleblind study. Acta Psychiatrica Scandinavica, 103(6), 435-440.

National Institute for Clinical Excellence 2004. Depression: Management of Depression in Primary and Secondary Care, Clinical Guideline 23, December, London. 
Nelson, J.C., Wohlreich, M.M., Mallinckrodt, C.H. et al. 2005. Duloxetine for the treatment of major depressive disorder in older people. American Journal of Geriatric Psychiatry, 13(3), 227-235.

Nierenberg, A.A., Feighner, J.P., Rudolph, R. et al. 1994. Venlafaxine for treatment-resistant unipolar depression. Journal of Clinical Psychopharmacology, 14, 419-423.

O'Brien, J. \& Barber, B. 2000. Neuroimaging in dementia and depression. Advances in Psychiatric Treatment, 6, 109-119.

Old Age Depression Interest Group 1993. How long should the elderly take antidepressants? A double-blind placebo-controlled study of continuation/prophylaxis therapy with dothiepin. British Journal of Psychiatry, 162, 75-182.

Oslin, D.W., Have, T.R.T. \& Streim, J.E. et al.2003. Probing the safety of medications in the frail elderly; evidence from a randomised clinical trial of sertraline and venlafaxine in depressed nursing home residents. Journal of Clinical Psychiatry, 64(8), 875-882.

Penninx, B.W., Deeg, D.J., van Eijk, J.T. et al. 2000. Changes in depression and physical decline in older adults; a longitudinal perspective. Journal of Affective Disorders, 61, 1-12.

Pridmore, S. \& Turnier, S.Y. 2004. Medication options in the treatment of treatment-resistant depression. Australian and New Zealand Journal of Psychiatry, 38(4), 219-225.

Rapaport,M.H., Schneider, L.S., Dunner, D.L. et al. 2003. Efficacy of controlled-release paroxetine in the treatment of late-life depression. Journal of Clinical Psychiatry, 64(9), 1065-1074.

Renwick, R.A. 1985. Treatment resistant depression. Psychiatric Journal of University of Ottawa, 46, 576-584.

Reynolds, C.F., III, Perel, J.M. \& Frank E, et al. 1999. Three-year outcomes of maintenance nortriptyline treatment in late-life depression: a study of two fixed plasma levels. American Journal of Psychiatry, 156(8), 1177-1181.

Roth, M., Montjoy, C. \& Amrein, R. 1996. Moclobemide in elderly patients with cognitive decline and depression: an international double-blind trial, placebo-controlled trial. British Journal of Psychiatry, 168(2), 149-157.

Schatzberg, A.F., Kremer, C., Rodrigues, H.E., Murphy \& G.M. et al. 2002. Double-blind, randomised comparison of mirtazapine and paroxetine in elderly depressed patients. American Journal of Geriatric Psychiatry, 10(5), 541-550.

Shapira, B., Tub,i N., Drexler, H., Lidsky, D., Calev, A. \& Lerer, B. 1998. Cost and benefit in the choice of ECT schedule. Twice versus three times weekly ECT. British Journal of Psychiatry, 172, 44-48. 
Sheikh, J..I, Cassidy, E.L., Doraiswamy, P.M. et al. 2004. Efficacy, safety and tolerability of sertraline in patients with late-life depression. Journal of the American Geriatrics Society, 52(1). 86-92.

Smith, D., Dempster, C., Glanvile, J. et al. 2002. Efficacy and tolerability of venlafaxine compared with selective serotonin reuptake inhibitors and other antidepressants; a meta-analysis. British Journal of Psychiatry, 180, 396404.

Stah,I S. \& Briley, M. 2004. Understanding pain in depression. Human Psychopharmacology, 19, S9-S13.

Stahl, S 2000. Essential Psychopharmacology; Neuroscientific Basis and Practical Applications. Second Edition, Cambridge, CUP.

Stahl, S.M. 1998. Basic psychopharmacology of antidepressants part 2: oestrogen as an adjunct to antidepressant treatment. Journal of Clinical Psychiatry, 59(4), 15-24.

Thase, M.E. \& Rush, A.J. 1997. Treatment resistant depression. In: Bloom FEaKD, editor. Psychopharmacology: The Fourth Generation of Progress. New York: Raven Press, 1081-1215.

Thase, M.E., Entsuah, R. \& Rudolph, R.L. 2001. Remission rates during treatment with venlafaxine or selective serotonin reuptake inhibitors. British Journal of Psychiatry, 178, 234-241.

Timmer, .CL., Paanakker, J.E. \& van Hal, H.J.M. 1996. Pharmacokinetics of mirtazapine from orally administered tablets: influence of age, gender and treatment regimen. Human Psychopharmacology, 11, 497-509.

Tourigny-Rivard, M.F. 1997. Pharmacotherapy of affective disorders in old age. Canadian Journal of Psychiatry, 42 (1), 10-18.

Trick, L., Neil, S., Una, R. et al. 2004. A double-blind, randomised, 26-week study comparing the cognitive and psychomotor effects and efficacy of 75 $\mathrm{mg}(37.5 \mathrm{mg}$ bd) venlafaxine and $25 \mathrm{mg} / 50 \mathrm{mg}$ of dothiepin in elderly patients with moderate major depression being treated in general practice. Journal of Psychopharmacology, 18(2), 205-214.

Williams, J.W., Mulrow, C.D, Chiquette E. et al. 2000. A systematic review of newer pharmacotherapies for depression in adults; evidence report summary. Annals of Internal Medicine, 132, 743-756.

Wilson, K., Mottram, P., Sivanranthan, A., Nightingale, A. 2001. Antidepressant versus placebo for depressed elderly (Cochrane Review). In: The Cochrane Library, 2001; Issue 2. Oxford: Update Software.

Wilson, K., Mottram, P., Sivanranthan, A. et al. 2004. Antidepressants versus placebo for the depressed elderly. Cochrane Review, 3, 2004.

Wilson, K.C.M. \& Copeland, J.R.M. \& Taylor, S. et al. 1999. Natural history of pharmacotherapy of older depressed community residents. British Journal of Psychiatry, 175, 439-443. 
Wilson, K.C.M., Mottram, P.G., Ashworth. L, \& Abou-Saleh, M.T. 2003. Older community residents with depression; long-term treatment with sertraline. British Journal of Psychiatry, 182, 492-497.

Yamashita, H. 2000. Clinical features and treatment response of patients with major depression and silent cerebral infarction. Neuropsychobiology 44(4), Nov-182. 
